\title{
Identification of key candidate tumor biomarkers in non-small-cell lung cancer by in silico analysis
}

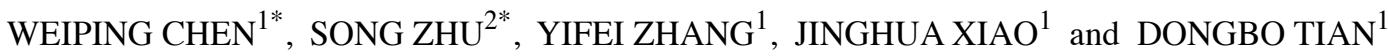 \\ Departments of ${ }^{1}$ Respiratory Medicine and ${ }^{2}$ Radiotherapy, The Sixth Affiliated Hospital of Guangzhou \\ Medical University, Qingyuan People's Hospital, Qingyuan, Guangdong 511518, P.R. China
}

Received November 29, 2018; Accepted November 12, 2019

DOI: $10.3892 / \mathrm{ol} .2019 .11169$

\begin{abstract}
Lung cancer is a common malignancy worldwide. The aim of the present study was to investigate differentially expressed genes (DEGs) between non-small-cell lung cancer (NSCLC) and normal lung tissue, and to reveal the potential molecular mechanism underlying NSCLC. The Gene Expression Omnibus database was used to obtain three gene expression profiles (GSE18842, GSE30219 and GSE33532). DEGs were obtained by GEO2R. Gene Ontology and pathway enrichment analyses were performed for DEGs in the Database for Annotation, Visualization and Integrated Discovery. A protein-protein interaction (PPI) network of DEGs was constructed and analyzed using the Search Tool for the Retrieval of Interacting Genes/Proteins database and Cytoscape software. A survival analysis was performed and protein expression levels of DEGs in human NSCLC were analyzed in order to determine clinical significance. A total of 764 DEGs were identified, consisting of 428 upregulated and 336 downregulated genes in NSCLC tissues compared with normal lung tissues, which were enriched in the 'cell cycle', 'cell adhesion molecules', 'p53 signaling pathway', 'DNA replication' and 'tight junction'. A PPI network of DEGs consisting of 51 nodes and 192 edges was constructed. The top 10 genes were identified as hub genes from the PPI network. High expression of 4 of the 10 hub genes was associated with worse overall survival rate in patients with NSCLC, including CDK1, PLK1, RAD51 and RFC4. In conclusion, the present study aids in improving the current understanding of aberrant gene expression between NSCLC tissues and normal lung tissues underlying tumorgenesis in NSCLC. Identified
\end{abstract}

Correspondence to: Professor Dongbo Tian, Department of Respiratory Medicine, The Sixth Affiliated Hospital of Guangzhou Medical University, Qingyuan People's Hospital, B24 Yinquan Road, Qingyuan, Guangdong 511518, P.R. China

E-mail: 444014503@qq.com

*Contributed equally

Key words: non-small-cell lung cancer, in silico analysis, differentially expressed genes hub genes can be used as a tumor marker for diagnosis and prognosis or as a drug therapy target in NSCLC.

\section{Introduction}

Lung cancer is the leading cause of cancer-associated mortality worldwide. In 2015, an estimated 221,200 new cases (115,610 in men and 105,590 in women) of lung and bronchial cancer were diagnosed, and 158,040 deaths (86,380 in men and 71,660 in women) were estimated to occur as a result of the disease worldwide (1). Following diagnosis, only $16.8 \%$ of all patients with lung cancer live beyond 5 years (2). This is primarily attributed to the lack of early effective diagnostic measures and high recurrence rates. Approximately $50 \%$ of patients are diagnosed with advanced lung cancer, whose 5-year survival rate is $<15 \%$ (3-5).

Currently, molecular biomarkers are used to diagnose lung cancer. ProGRP, SCC-Ag, Cyfra21-1 and CEA are widely used as lung cancer serum biomarkers (6). However, a meta-analysis study reported that the sensitivity levels of ProGRP, SCC-Ag, Cyfra21-1 and CEA in the serum of patients with lung cancer were $<60 \%$ (6). Thus, investigating the molecular mechanism underlying tumorigenesis, and discovering new biomarkers can help improve diagnosis. In recent years, a number of high-throughput platforms, such as microarray technology, have been widely used to study gene expression during tumorigenesis. Now, a new approach combined with microarray technology and bioinformatics analysis allows the comprehensive analysis of gene expression changes in non-small cell lung cancer (NSCLC) (7-9).

In the present study, taking into account the microarray results of false positives, three mRNA microarray datasets were analyzed in order to investigate differentially expressed genes (DEGs) between NSCLC and normal tissue. Gene Ontology (GO) and pathway enrichment analysis were combined in order to identify functional DEGs, followed by protein interaction and survival analysis to identify hub genes in NSCLC.

\section{Materials and methods}

Microarray data. The Gene Expression Omnibus (GEO; http://www.ncbi.nlGSE18842m.nih.gov/geo) is a public repository for the storage of data, such as microarray and 
next-generation sequencing data, which is freely available to users. The GEO database was used to obtain three gene expression profiles. GSE18842, GSE30219 and GSE33532 (10-12) were obtained from the GEO database. Experiments with the selected three datasets were performed in the Affymetrix Human Genome U133 Plus 2.0 Array microarray platform (GPL570; version 2.0; Affymetrix; Thermo Fisher Scientific, Inc.).

Identification of DEGs. GEO2R (13) is an online interactive network tool that allows users to compare two or more sets of samples in order to identify the DEGs in a GEO data series. The results are presented as a table of genes ordered by significance. The present study used GEO2R to screen DEGs between NSCLC and normal lung samples. The adjusted P-values (adj. P) were used to correct the occurrence of false positive results. The adj. $\mathrm{P}<0.01$ and $\mid \log \mathrm{FCl}>1$ were set as the cut-off criterion as an indicator of significance.

GO and Kyoto Encyclopedia of Genes and Genomes (KEGG) pathway enrichment analysis. The Database for Annotation, Visualization and Integrated Discovery (DAVID; version 6.7; http://david.abcc.ncifcrf.gov) is an online interactive tool that provides a comprehensive set of functional annotation tools for researchers to understand the biological meaning behind numerous different genes (7). The GO and KEGG pathway enrichment analysis panels in the DAVID were applied in order to identify DEGs. $\mathrm{P}<0.05$ was set as the cut-off criterion for indicating significance.

Protein-protein interaction (PPI) network construction and module selection. The Search Tool for the Retrieval of Interacting Genes (STRING; http://string.embl.de) database was applied to construct a PPI network of DEGs (14). The confidence score $\geq 0.4$ was set as the cut-off criterion. Subsequently, the Molecular Complex Detection (MCODE) panel in the Cytoscape software (version 3.7.2) was applied to screen significant modules in the PPI network (15). The degree cutoff $=2$, node score cutoff $=0.2, k$-core $=2$, and $\max$. depth=100 were set as the cut-off criterion (16). The functional enrichment analysis of genes in the selected module was also performed by KEGG and GO panels in the DAVID.

Survival analysis and protein expression in human NSCLC. Kaplan-Meier plotter (2018 version; http://kmplot.com/analysis) is an online, meta-analysis-based web tool that is used for biomarker assessment. The tool is capable of assessing the effect of 54,675 genes on survival rate using 10,461 cancer samples. The present study used this online tool to investigate the prognostic value of DEGs for patients with NSCLC in a large public clinical microarray database (http://kmplot. com/analysis/index.php?p=service\&cancer=lung) (17).

Protein expression in NSCLC tissues and normal lung tissues was determined from The Human Protein Atlas (2018 version, www.proteinatlas.org).

\section{Results}

Identification of DEGs. GSE18842 included 46 NSCLC samples and 45 normal samples. GSE30219 consisted of 229

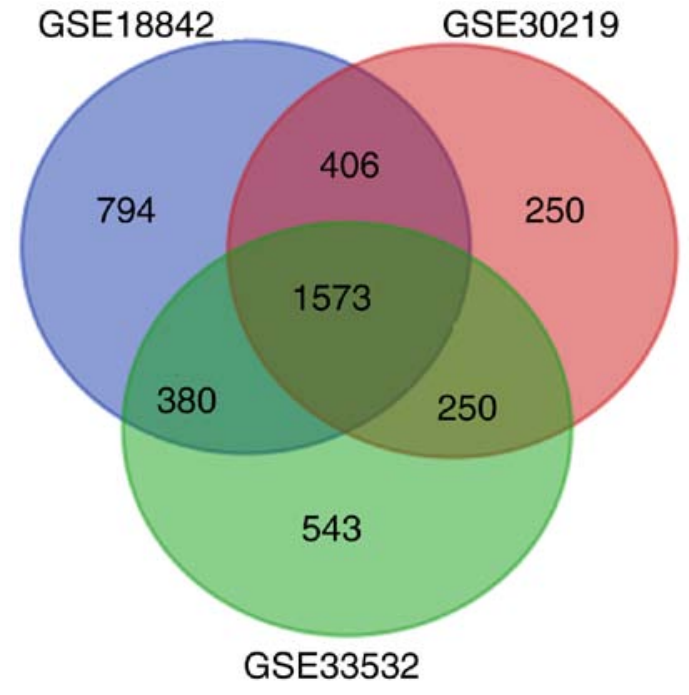

Figure 1. Identification of differentially expressed genes in three expression profiling datasets GSE18842, GSE30219 and GSE33532.

NSCLC cancer samples and 14 healthy lung samples. The array data of GSE33532 included 80 NSCLC tissue samples and 20 normal samples. All samples were confirmed by histopathology. Based on the GEO2R analysis, a total of 3,153, 2,479 and 2,746 DEGs were identified from the GSE18842, GSE30219 and GSE33532 datasets, respectively. A total of 1,573 genes were screened out by taking an intersection of all three GEO datasets (Fig. 1). Among them, 764 genes exhibited the same trend in expression, consisting of 428 upregulated and 336 downregulated genes in NSCLC tissues compared with normal lung tissues (Table SI).

GO and KEGG pathway enrichment analysis. In order to further determine the function of DEGs, the present study used the DAVID for the functional and pathway enrichment analysis. The GO analysis revealed that upregulated DEGs were primarily involved in ' $\mathrm{M}$ phase' and 'cell cycle phase', while downregulated DEGs were primarily involved in 'plasma membrane part', and 'vasculature development'. Notably, the KEGG pathways analysis demonstrated that upregulated DEGs were enriched in the 'cell cycle', 'p53 signaling pathway' and 'DNA replication', (Fig. 2A and Table I) while downregulated DEGs were enriched in 'vascular smooth muscle contraction', 'cell adhesion molecules' and 'tight junction' (Fig. 2B and Table I). Overall, a total of 115 genes were enriched, including 68 upregulated DEGs and 47 downregulated DEGs (Table SII). The 115 genes were used for further PPI analysis.

PPI network construction and modules selection. The PPI network of DEGs consisted of 51 nodes and 192 edges, including 33 upregulated and 18 downregulated genes (Fig. 3A). Degrees $\geq 10$ were set as the cut-off criterion (Table SIII). The top 10 genes were selected as hub genes, including cyclin-dependent kinase 1 (CDK1), checkpoint kinase 1 (CHEK1), budding uninhibited by benzimidazoles 1 (BUB1), replication factor C 4 (RFC4), polo-like kinase 1 (PLK1), RAD51 recombinase (RAD51), minichromosome maintenance complex component (MCM) 2, MCM4, MLF1 interacting protein (MLF1IP) and MCM6; all of which were upregulated. Furthermore, the most 
A

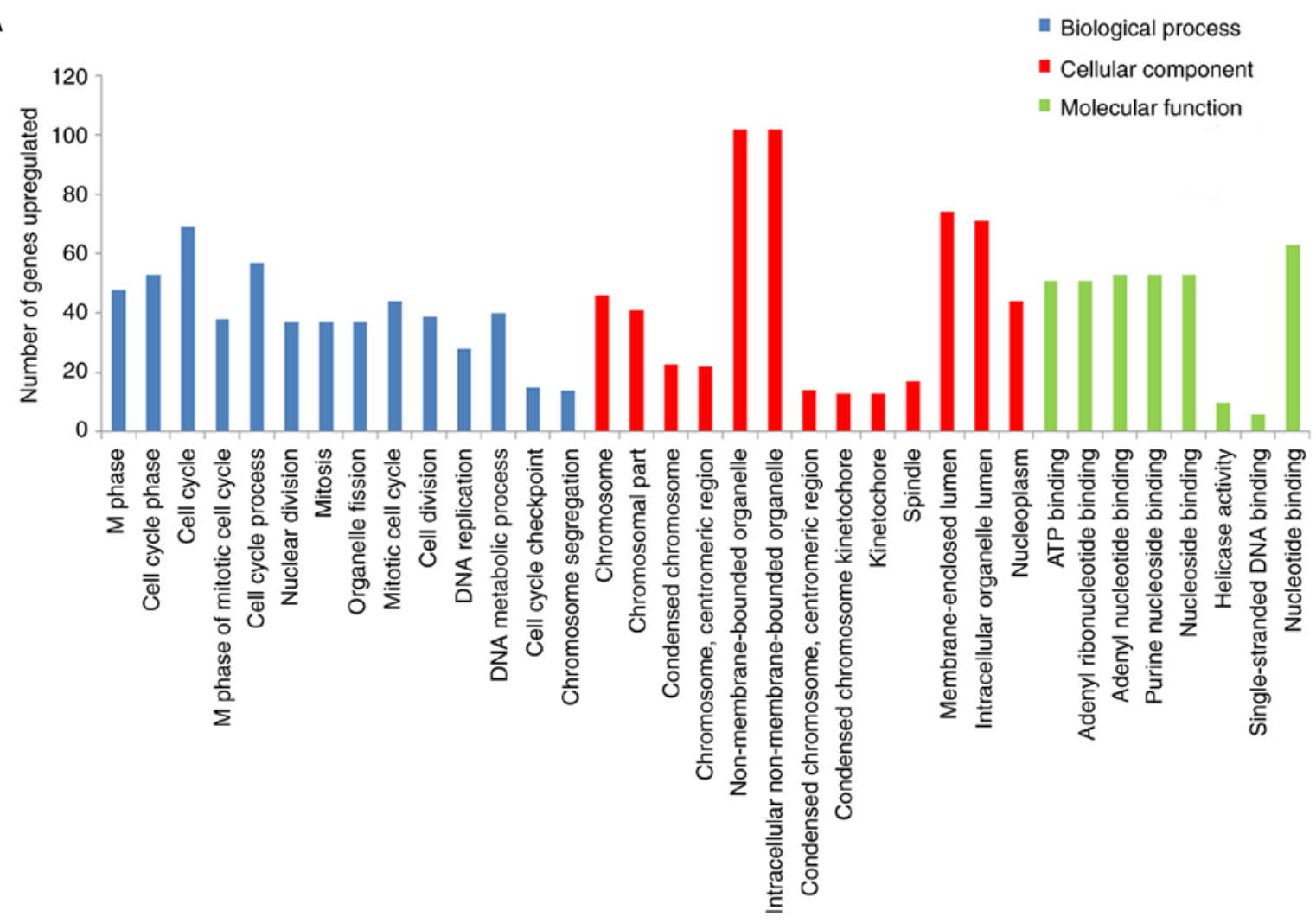

B



Figure 2. Enriched GO terms of differentially expressed genes in non-small cell lung cancer. DEGs were classified by GO analysis into three groups including biological process (blue), cellular component (red) and molecular function (green). (A) The number of genes upregulated for each functional classification. (B) The number of genes downregulated for each functional classification.

significant module was identified from the PPI network using the MCODE app, including 14 nodes and 85 edges (Fig. 3B). The GO and KEGG pathway enrichment analyses revealed that genes in this module were significantly associated with 'mitotic cell cycle', 'DNA unwinding involved in DNA replication' and 'DNA replication pathway' (Table II). 
Table I. GO and KEGG pathway enrichment analysis of upregulated and downregulated genes.

(A) Upregulated

\begin{tabular}{|c|c|c|c|c|}
\hline Term & Function & Gene count & $\%$ & P-value \\
\hline GO:0000279 & M phase & 48 & 12.8 & $1.00 \times 10^{-24}$ \\
\hline GO:0022403 & Cell cycle phase & 53 & 14.1 & $1.30 \times 10^{-24}$ \\
\hline GO:0007049 & Cell cycle & 69 & 18.4 & $5.70 \times 10^{-23}$ \\
\hline GO:0000087 & $\mathrm{M}$ phase of mitotic cell cycle & 38 & 10.1 & $8.10 \times 10^{-22}$ \\
\hline GO:0022402 & Cell cycle process & 57 & 15.2 & $2.10 \times 10^{-21}$ \\
\hline GO:0005694 & Chromosome & 46 & 12.3 & $2.20 \times 10^{-17}$ \\
\hline GO:0044427 & Chromosomal part & 41 & 10.9 & $2.20 \times 10^{-16}$ \\
\hline GO:0000793 & Condensed chromosome & 23 & 6.1 & $8.10 \times 10^{-14}$ \\
\hline GO:0000775 & Chromosome, centromeric region & 22 & 5.9 & $3.50 \times 10^{-13}$ \\
\hline GO:0043228 & Non-membrane-bounded organelle & 102 & 27.2 & $1.20 \times 10^{-9}$ \\
\hline GO:0005524 & ATP binding & 51 & 13.6 & $3.00 \times 10^{-4}$ \\
\hline GO:0032559 & Adenyl ribonucleotide binding & 51 & 13.6 & $4.10 \times 10^{-4}$ \\
\hline GO:0030554 & Adenyl nucleotide binding & 53 & 14.1 & $4.20 \times 10^{-4}$ \\
\hline GO:0001883 & Purine nucleoside binding & 53 & 14.1 & $6.00 \times 10^{-4}$ \\
\hline GO:0001882 & Nucleoside binding & 53 & 14.1 & $7.00 \times 10^{-4}$ \\
\hline KEGG:hsa04110 & Cell cycle & 20 & 5.3 & $7.70 \times 10^{-11}$ \\
\hline KEGG:hsa04115 & p53 signaling pathway & 11 & 2.9 & $4.20 \times 10^{-6}$ \\
\hline KEGG:hsa03030 & DNA replication & 7 & 1.9 & $1.90 \times 10^{-4}$ \\
\hline KEGG:hsa00670 & One carbon pool by folate & 5 & 1.3 & $4.70 \times 10^{-4}$ \\
\hline KEGG:hsa04114 & Oocyte meiosis & 8 & 2.1 & $1.70 \times 10^{-2}$ \\
\hline
\end{tabular}

(B) Downregulated

\begin{tabular}{|c|c|c|c|c|}
\hline Term & Function & Gene count & $\%$ & P-value \\
\hline GO:0044459 & Plasma membrane part & 84 & 27.5 & $1.70 \times 10^{-11}$ \\
\hline GO:0005886 & Plasma membrane & 117 & 38.4 & $1.40 \times 10^{-10}$ \\
\hline GO:0001944 & Vasculature development & 23 & 7.5 & $2.10 \times 10^{-10}$ \\
\hline GO:0001568 & Blood vessel development & 22 & 7.2 & $8.40 \times 10^{-10}$ \\
\hline GO:0048514 & Blood vessel morphogenesis & 19 & 6.2 & $1.50 \times 10^{-8}$ \\
\hline GO:0007155 & Cell adhesion & 32 & 10.5 & $6.40 \times 10^{-7}$ \\
\hline GO:0022610 & Biological adhesion & 32 & 10.5 & $6.60 \times 10^{-7}$ \\
\hline GO:0003779 & Actin binding & 20 & 6.6 & $2.20 \times 10^{-6}$ \\
\hline GO:0008092 & Cytoskeletal protein binding & 24 & 7.9 & $1.10 \times 10^{-5}$ \\
\hline GO:0050431 & Transforming growth factor beta binding & 5 & 1.6 & $1.40 \times 10^{-5}$ \\
\hline GO:0031226 & Intrinsic to plasma membrane & 44 & 14.4 & $2.20 \times 10^{-5}$ \\
\hline GO:0005887 & Integral to plasma membrane & 42 & 13.8 & $6.20 \times 10^{-5}$ \\
\hline GO:0009986 & Cell surface & 19 & 6.2 & $8.20 \times 10^{-5}$ \\
\hline GO:0051015 & Actin filament binding & 7 & 2.3 & $2.40 \times 10^{-4}$ \\
\hline GO:0032403 & Protein complex binding & 12 & 3.9 & $4.40 \times 10^{-4}$ \\
\hline KEGG:hsa04270 & Vascular smooth muscle contraction & 9 & 3.0 & $1.20 \times 10^{-3}$ \\
\hline KEGG:hsa04514 & Cell adhesion molecules & 9 & 3.0 & $3.50 \times 10^{-3}$ \\
\hline KEGG:hsa05414 & Dilated cardiomyopathy & 7 & 2.3 & $7.90 \times 10^{-3}$ \\
\hline KEGG:hsa04530 & Tight junction & 8 & 2.6 & $1.40 \times 10^{-2}$ \\
\hline KEGG:hsa05410 & Hypertrophic cardiomyopathy & 6 & 2.0 & $2.20 \times 10^{-2}$ \\
\hline
\end{tabular}

GO, Gene Ontology; KEGG, Kyoto Encyclopedia of Genes and Genomes; has, homosapien.

Survival analysis and protein expression in human NSCLC. The degree cutoff $>16$, node score cutoff $=0.2$, $\mathrm{k}$-core $=2$, and max.depth $=100$ were set as the cut-off criterion for PPI analysis, applied to predict PPI network of DEGs. The 6 genes were selected as core hub genes, including CDK1, CHEK1, BUB1, RFC4, PLK1 and RAD51. The prognostic value of the hub genes was evaluated using Kaplan-Meier plotter. The overall survival rate analysis demonstrated that high expression of 


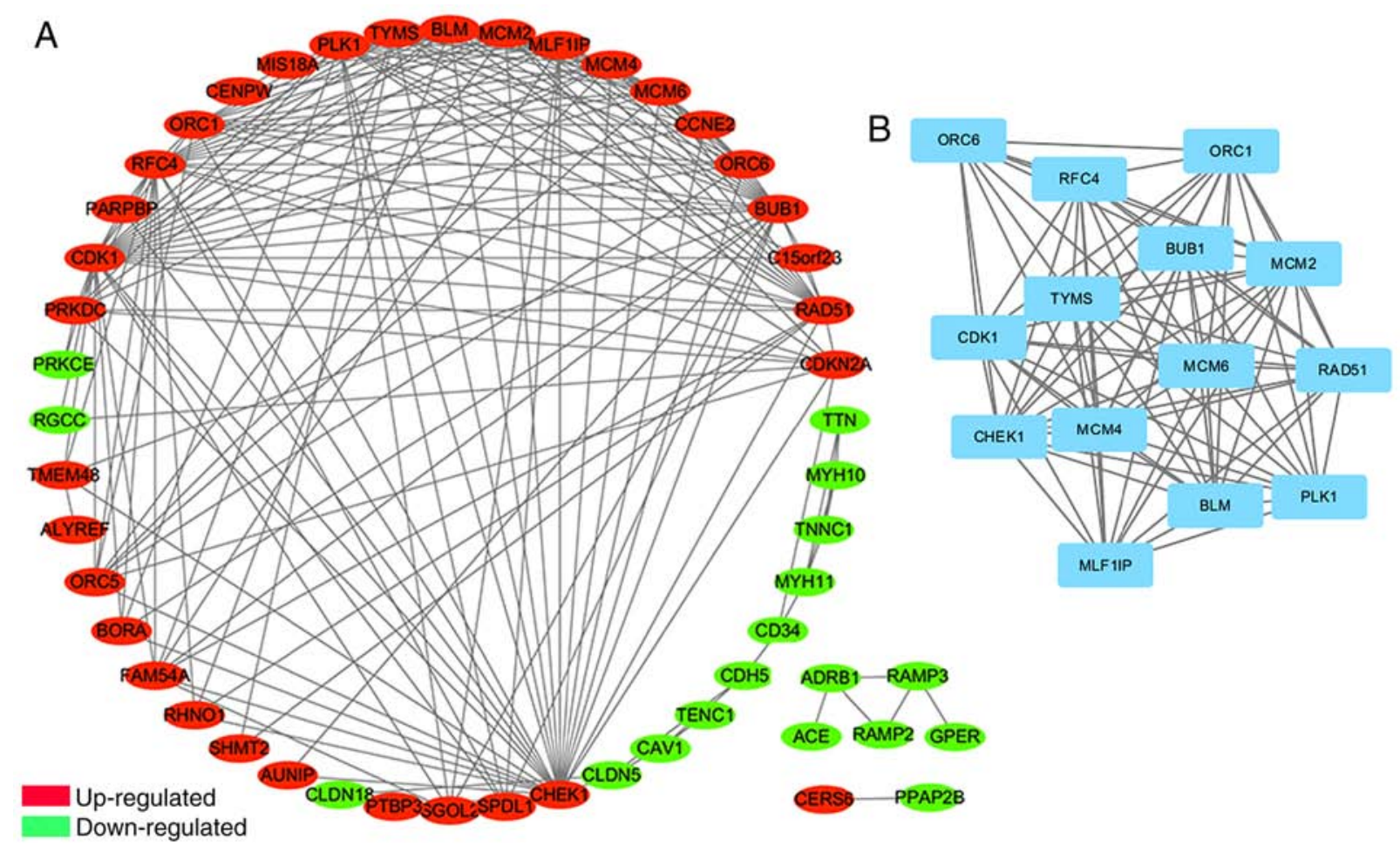

Figure 3. PPI network of DEGs and modular analysis. (A) The Search Tool for the Retrieval of Interacting Genes/Proteins database was applied to predict the PPI network of DEGs. The PPI network of DEGs consisted of 51 nodes and 192 edges, including 33 upregulated genes and 18 downregulated genes. (B) The most significant module from the PPI network, including 14 nodes and 85 edges. All genes in this module were upregulated. PPI, protein-protein interaction; DEGs, differentially expressed genes.

CDK1 [hazard ratio (HR), 1.84; $95 \%$ confidence interval (CI), 1.62-2.10; $\mathrm{P}<1 \times 10^{-16} ;$ Fig. $\left.4 \mathrm{~A}\right]$ caused the low overall survival rate for NSCLC, which was the same as PLK1 (HR, 1.66; 95\% CI, 1.46-1.89; P=3.3×10-15; Fig. 4B), RAD51 (HR, 1.84; 95\% CI, 1.62-2.09; P<1x10-16; Fig. 4C), RFC4 (HR, 1.73; 95\% CI, 1.52-1.97; P<1 $\times 10^{-16}$ Fig. 4D), BUB1 (HR, 1.21; 95\% CI, 1.07-1.38; $\mathrm{P}=0.0025$; data not shown), but not CHEK1 (HR, 1.42; 0.99-2.04; $\mathrm{P}=0.052$; data not shown). HR $>1.5$ and $\mathrm{P}<0.05$ were set as the cut-off criterion for the survival analysis. According to these cut-off criteria, four genes were identified as potential tumor markers for NSCLC, including CDK1, PLK1, RAD51 and RFC4. In order to determine the clinical relevance of hub gene expression, the present study then analyzed the expression of proteins from clinical specimens in The Human Protein Atlas database. The database indicated that CDK1 $\left(\mathrm{P}=1.03 \times 10^{-3}\right)$ was highly expressed in NSCLC compared with the low expression observed in normal lung samples, which was also true for PLK1 $\left(\mathrm{P}=4.73 \times 10^{-9}\right)$, RAD51 $\left(\mathrm{P}=2.93 \times 10^{-3}\right)$ and RFC4 $\left(\mathrm{P}=7.27 \times 10^{-4}\right)$ (Fig. 5).

\section{Discussion}

The development of NSCLC is a multi-step process that involves interactions between genetic, epigenetic aberrations and environmental factors, which leads to disorders of key oncogenes and tumor repressors $(1,18)$. Knowledge of the molecular mechanism underlying NSCLC is essential for diagnosis and treatment. The development of microarrays and high throughput sequencing techniques that can simultaneously detect mRNA expression levels of thousands of genes has benefited the prediction of potential diagnostic and therapeutic target genes for NSCLC (10). The present study extracted data from three gene expression profiles, GSE18842, GSE30219 and GSE33532. A total of 428 upregulated and 336 downregulated genes were identified between NSCLC samples and normal lung tissues. GO and KEGG annotations revealed that DEGs were enriched in the 'cell cycle', 'cell adhesion molecules' and 'tight junction'. Further PPI analysis, survival analysis and The Human Protein Atlas identified 4 hub genes that can be used as a tumor marker for diagnosis and prognosis or as a drug therapy target in NSCLC.

GEO2R (13) is an online interactive network tool used to identify the DEGs in GEO datasets. In the present study, a total of 764 DEGs were screened out between NSCLC samples and normal lung tissues with the GEO2R analysis, consisting of 428 upregulated and 336 downregulated genes. The GO and KEGG functional annotations revealed that upregulated DEGs were enriched in the 'cell cycle', 'p53 signaling pathway' and 'DNA replication', while downregulated DEGs were enriched in 'vascular smooth muscle contraction', 'cell adhesion molecules' and 'tight junction'. In accordance with Singhal et al (19), Voortman et al (20) reported that an imbalance of $\mathrm{G}_{2}-\mathrm{M}$-phase arrest in the cell cycle can lead to the occurrence of NSCLC, which is one of its primary causes (21). Furthermore, the majority of NSCLC cases have p53 mutations and, as a result, an imbalanced expression of p53 target genes, such as p21, Bax and PUMA, which ultimately prompts the growth of tumor cells (22).

A total of 10 genes that had a high degree in the PPI network were selected as hub genes. The top 10 degree hub genes were as follows: CDK1, CHEK1, BUB1, RFC4, PLK1, 
Table II. Functional and pathway enrichment analysis of genes in the module.

\begin{tabular}{|c|c|c|c|c|}
\hline Pathway ID & Pathway description & Gene count & $\begin{array}{c}\text { False } \\
\text { discovery rate }\end{array}$ & Nodes \\
\hline GO.0000278 & Mitotic cell cycle & 12 & $8.58 \times 10^{-12}$ & $\begin{array}{l}\text { BLM, BUB1, CHEK1, MCM2, MCM4, } \\
\text { MCM6, MLF1IP, ORC1, ORC6, PLK1, } \\
\text { RFC4, TYMS }\end{array}$ \\
\hline GO.0044772 & $\begin{array}{l}\text { Mitotic cell cycle phase } \\
\text { transition }\end{array}$ & 9 & $2.87 \times 10^{-10}$ & $\begin{array}{l}\text { CDK1, CHEK1, MCM2, MCM4, MCM6, } \\
\text { ORC1, ORC6, PLK1, TYMS }\end{array}$ \\
\hline GO.1903047 & Mitotic cell cycle process & 10 & $5.38 \times 10^{-9}$ & $\begin{array}{l}\text { BLM, BUB1, CHEK1, MCM2, MCM4, } \\
\text { MCM6, ORC1, ORC6, PLK1, TYMS }\end{array}$ \\
\hline GO.0000082 & $\begin{array}{l}\mathrm{G}_{1} / \mathrm{S} \text { transition of mitotic } \\
\text { cell cycle }\end{array}$ & 7 & $1.74 \times 10^{-8}$ & $\begin{array}{l}\text { CDK1, MCM2, MCM4, MCM6, ORC1, } \\
\text { ORC6, TYMS }\end{array}$ \\
\hline GO.0006268 & $\begin{array}{l}\text { DNA unwinding involved in } \\
\text { DNA replication }\end{array}$ & 4 & $5.66 \times 10^{-8}$ & MCM2, MCM4, MCM6, RAD51 \\
\hline GO.0005524 & ATP binding & 11 & $9.36 \times 10^{-8}$ & $\begin{array}{l}\text { BLM, BUB1, CDK1, CHEK1, MCM2, } \\
\text { MCM4, MCM6, ORC1, PLK1, RAD51, } \\
\text { RFC4 }\end{array}$ \\
\hline GO.0000166 & Nucleotide binding & 12 & $1.62 \times 10^{-7}$ & $\begin{array}{l}\text { BLM, BUB1, CDK1, CHEK1, MCM2, } \\
\text { MCM4, MCM6, ORC1, PLK1, RAD51, } \\
\text { RFC4, TYMS }\end{array}$ \\
\hline GO.0043168 & Anion binding & 12 & $2.27 \times 10^{-7}$ & $\begin{array}{l}\text { BLM, BUB1, CDK1, CHEK1, MCM2, } \\
\text { MCM4, MCM6, ORC1, PLK1, RAD51, } \\
\text { RFC4, TYMS }\end{array}$ \\
\hline GO.0003697 & Single-stranded DNA binding & 4 & $3.14 \times 10^{-5}$ & BLM, MCM4, MCM6, RAD51 \\
\hline GO.0005654 & Nucleoplasm & 14 & $6.79 \times 10^{-10}$ & $\begin{array}{l}\text { BLM, BUB1, CDK1, CHEK1, MCM2, } \\
\text { MCM4, MCM6, MLF1IP, ORC1, ORC6, } \\
\text { PLK1, RAD51, RFC4, TYMS }\end{array}$ \\
\hline GO.0044454 & Nuclear chromosome part & 7 & $8.90 \times 10^{-7}$ & $\begin{array}{l}\text { BLM, BUB1, MCM2, ORC1, ORC6, } \\
\text { PLK1, RAD51 }\end{array}$ \\
\hline GO.0000228 & Nuclear chromosome & 7 & $1.45 \times 10^{-6}$ & $\begin{array}{l}\text { BLM, BUB1, CHEK1, MCM2, ORC1, } \\
\text { ORC6, PLK1 }\end{array}$ \\
\hline KEGG:hsa04110 & Cell cycle & 8 & $9.91 \times 10^{-13}$ & $\begin{array}{l}\text { BUB1, CHEK1, MCM2, MCM4, MCM6, } \\
\text { ORC1, ORC6, PLK1 }\end{array}$ \\
\hline KEGG:hsa03030 & DNA replication & 4 & $1.02 \times 10^{-6}$ & MCM2, MCM4, MCM6, RFC4 \\
\hline
\end{tabular}

GO, Gene Ontology; KEGG, Kyoto Encylopedia of Genes and Genomes; has, homosapien.

RAD51, MCM2, MCM4, MLF1IP and MCM6. All these genes are upregulated in NSCLC. The GO and KEGG analyses revealed that the top 10 genes were enriched in 'mitotic cell cycle transition', 'ATP binding', and 'DNA replication'. It has been reported that the cell cycle checkpoint facilitated cellular responses to DNA damage, and an aberrant cell cycle facilitated the risk of cancer developing (23).

Survival analysis of the 10 genes revealed that selected hub genes were significantly associated with worse overall survival rate in patients with NSCLC, including CDK1, PLK1, RAD51 and RFC4. CDK1 is a member of the Ser/Thr protein kinase family (24). CDK1 was a master regulator of mitosis and meiosis, as a SUMO target both in vivo and in vitro involved in the initiation and transformation process through mitosis of the cell cycle (25). A number of studies have demonstrated that CDK1 inhibitors can block cell cycle progression through blocking mitosis and also have the potential to treat cancer due to their ability to control cell proliferation or inhibit tumor growth $(26,27)$. PLK1 belongs to the CDC5/Polo subfamily and is a Ser/Thr protein kinase (28). PLK1 is highly expressed during mitosis. PLK1 promotes cell proliferation and has also been observed to be upregulated in different types of human cancer. The deletion of PLK1 in cancer cells significantly inhibits cell proliferation and induces apoptosis (28). RAD51 was another selected hub gene. RAD51 is known to be involved in the homologous recombination and repair of DNA by interacting with the single stranded DNA-binding protein RPA and RAD52 (29). RAD51 is also involved in promoting tumorigenesis through interacting with BRCA1 and BRCA2, which are tumor suppressors (30-32). RFC4 is a member of the RFC family, which functions as a clamp loader that loads PCNA onto DNA and is involved in DNA repair activities $(33,34)$. Xiang et al $(35)$ reported that RFC4 is upregulated in patients with colorectal cancer, which could predict its prognosis as it promotes cell proliferation and cell cycle arrest. 
A

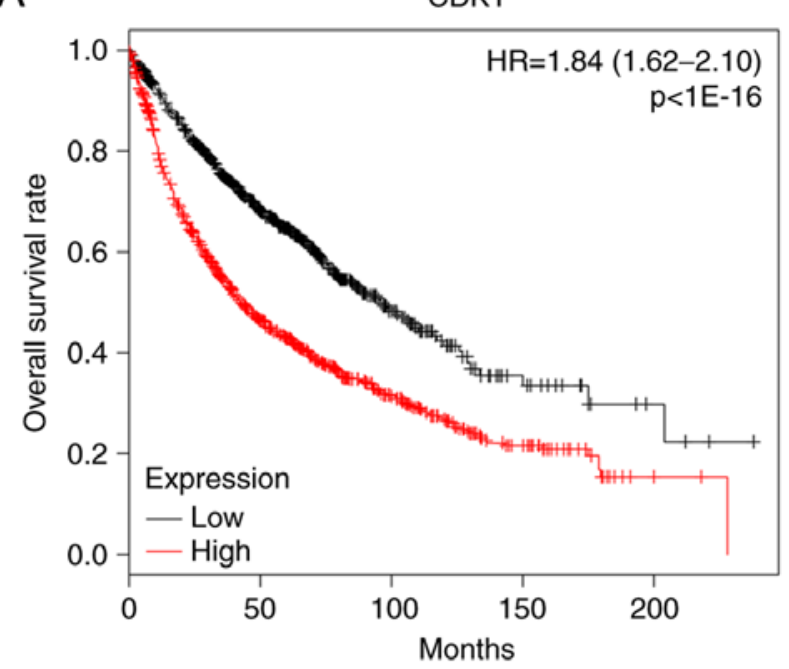

C

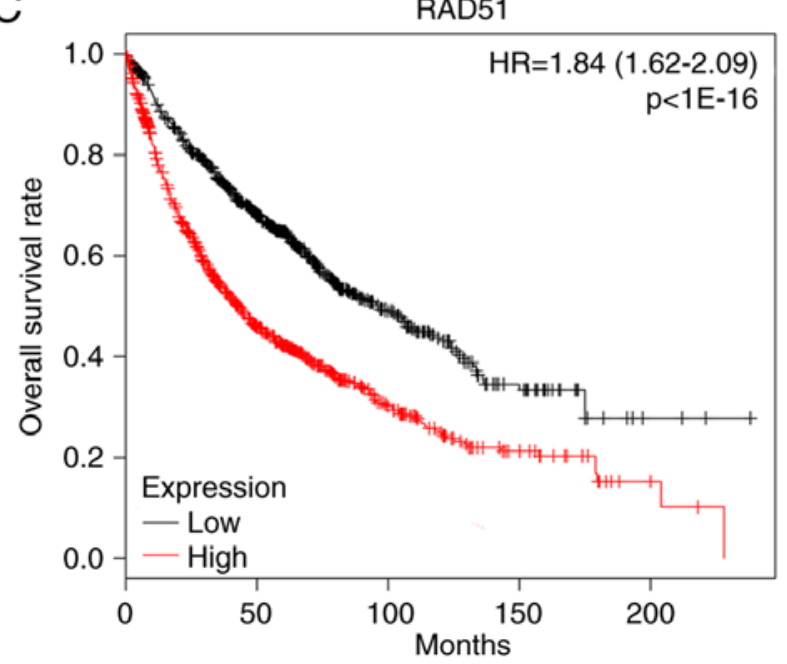

B

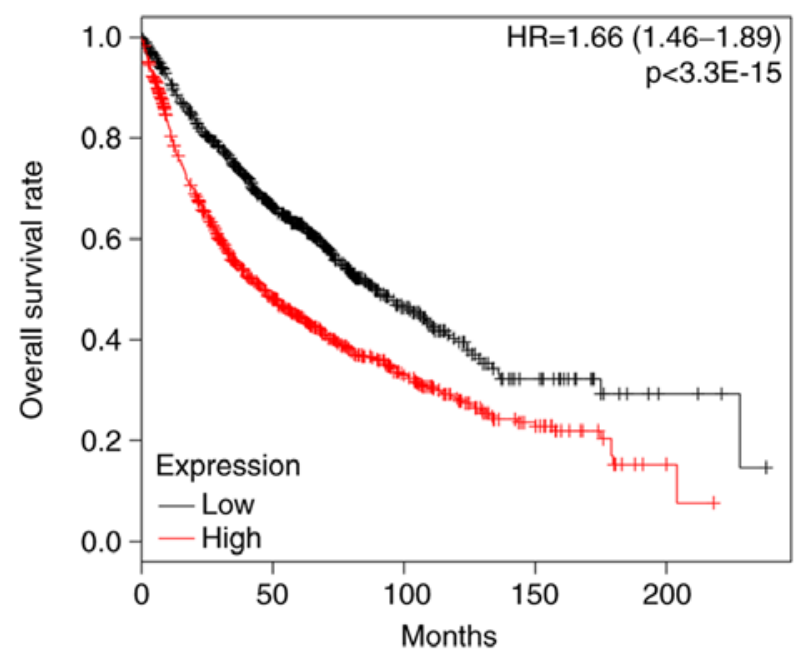

D

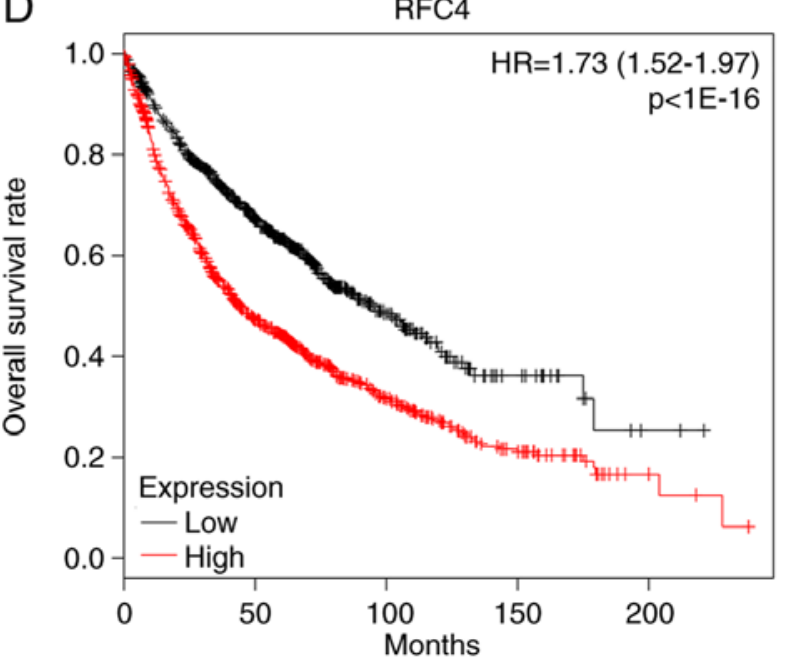

Figure 4. Prognostic value of hub genes in patients with NSCLC was investigated using a Kaplan-Meier plotter, assessing the overall survival rate of patients with NSCLC in specified hub gene ${ }^{\text {low }}$ and gene ${ }^{\text {high }}$ groups. The hub genes included (A) CDK1, (B) PLK1, (C) RAD51 and (D) RFC4. The Kaplan-Meier survival analysis revealed that patients with NSCLC that exhibited high expression levels of CDK1, PLK1, RAD51 and RFC4 had shorter overall survival rates. NSCLC, non-small cell lung carcinoma; HR, hazard ratio.

Due to the different selection methods and samples, the results of the present study were different from those of previous studies, which used the same lung cancer gene expression profiles (GSE18842, GSE30219 and GSE33532). Sanchez-Palencia et al (10) reported that KRT15 and PKP1, which may be good markers to distinguish squamous-cell carcinoma samples in GSE18842 (10). Rousseaux et al (11) reported that EBI3, PIWIL1, TPTE and NBPF4 may be potential biomarkers in lung cancer using the GSE30219 dataset (11). Meister et al (12) also reported that COL4A3, COL4A4 and CHRDL1 may be associated with lung cancer after analyzing the GSE33532 dataset (12). The present study revealed that four hub genes were significantly associated with worse overall survival of patients with NSCLC, including CDK1, PLK1, RAD51 and RFC4. To the best of our knowledge, RFC4 has not been reported as involved in the development of lung cancer before. RFC4 is involved in DNA replication as a clamp loader (35). In the present study, the results revealed that PLK1 and RFC4 were upregulated in NSCLC and were present in the cell cycle pathway, suggesting that the two genes may be important in the progression of NSCLC via the cell cycle pathway. Therefore, further experimental verification is required.

Overall, the present study provided a new comprehensive bioinformatics analysis to identify DEGs. The screened DEGs, including CDK1, PLK1, RAD51 and RFC4, can be used as tumor biomarkers for the diagnosis and prognosis, or as a drug therapy target, in NSCLC. However, further molecular biology experiments are required in order to confirm the underlying molecular mechanism of the genes identified in NSCLC.

There is a limitation to the present study. Heterogeneity within tumor cell populations is commonly observed in the majority of different types of cancer, particularly in lung cancer (36), which affects tumor growth rate, invasion and metastasis, and drug sensitivity and prognosis (37). NSCLC histopathology for the present study was confirmed by conventional paraffin-embedded tissue section, and the control samples were all from normal lung tissues, but not 

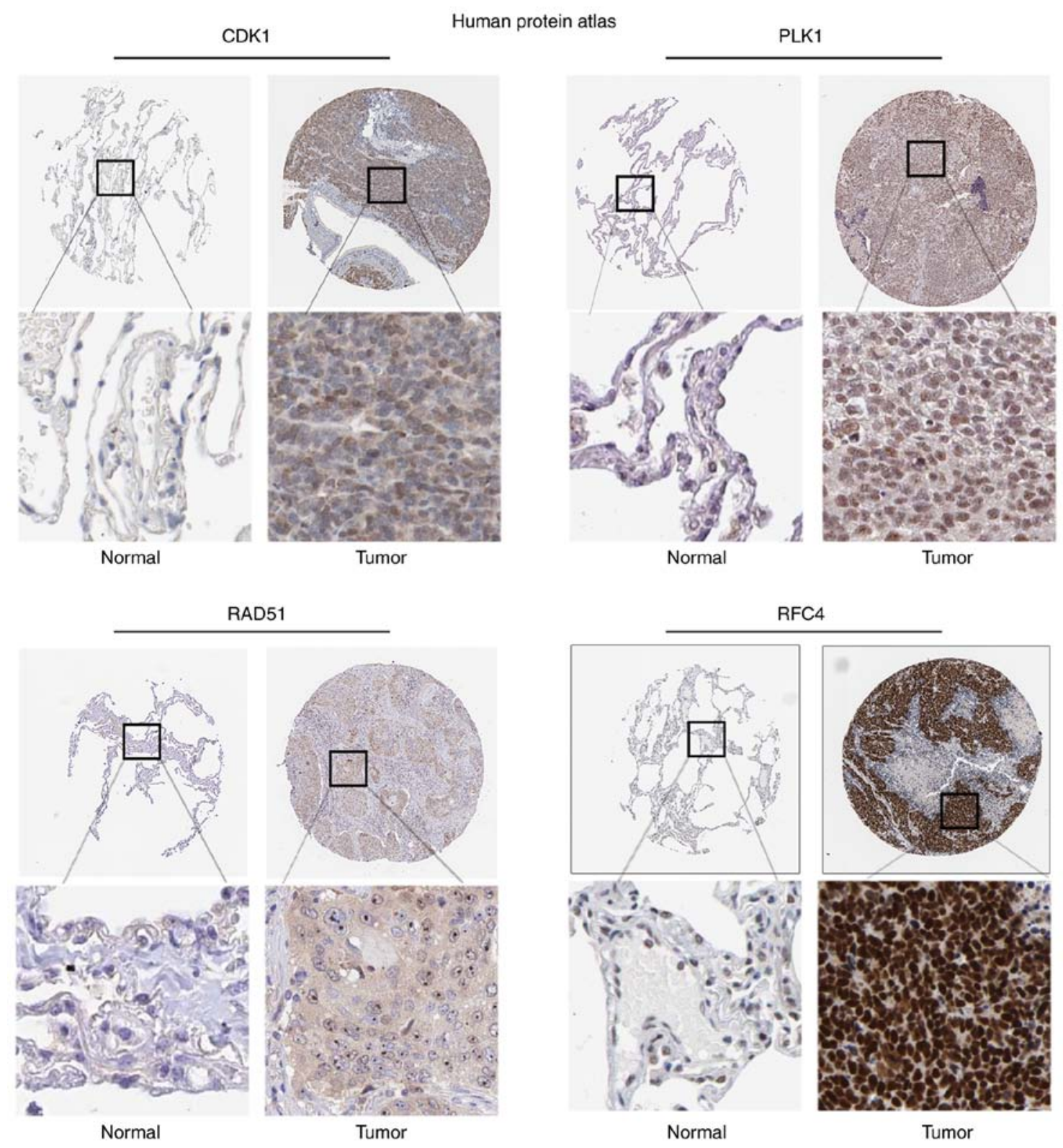

Figure 5. Hub gene protein expression in human NSCLC specimens was determined from The Human Protein Atlas. Representative IHC images of hub gene protein expression in NSCLC tissues and normal lung tissues. Each lower panel is a enlargement of the outlined area in the top panel in its respective column in the same sample. The IHC analysis demonstrated that CDK1 was highly expressed in NSCLC compared with that in normal lung samples, which was also true for PLK1, RAD51 and RFC4. The IHC images were downloaded from The Human Protein Atlas. NSCLC, non-small cell lung cancer; IHC, immunohistochemistry.

from their corresponding adjacent non-tumorous lung tissues. Fend et al (38) reported that the intrinsic heterogeneity of primary tissues in conventional paraffin sectioning with a mixture of various reactive cell populations can influence the results and interpretation of molecular studies. The authors also stated that a new technology called laser capture microdissection (LCM), is able to solve this problem (38). LCM is a powerful tool for isolating and studying the gene expression patterns of desired cells or tissues from heterogeneous populations (38). Isolation of a specific NSCLC cell from a heterogeneous tissue helps to obtain more meaningful molecular analysis results. Overall, the data from the present study suggested that data mining and integration analysis may be a useful tool for predicting cancer progression and understanding the molecular mechanisms underlying tumorgenesis.

\section{Acknowledgements}

Not applicable.

\section{Funding}

The present study was supported by the Medical Science and Technology Research Foundation of Guangdong Province (grant no. B2018217).

\section{Availability of data and materials}

The datasets generated and/or analyzed during the current study are available in the GEO repository, (https://www.ncbi. nlm.nih.gov/geo). 


\section{Authors' contributions}

WC, SZ conceived and designed the study. YZ, DT and JX performed the data analysis. WC and SZ wrote the article. All authors read and approved the final manuscript.

\section{Ethics approval and consent to participate}

Not applicable.

\section{Patient consent for publication}

Not applicable.

\section{Competing interests}

The authors declare that they have no competing interests.

\section{References}

1. Ansari J, Shackelford RE and El-Osta H: Epigenetics in non-small cell lung cancer: From basics to therapeutics. Transl Lung Cancer Res 5: 155-171, 2016.

2. Ramshankar V and Krishnamurthy A: Lung cancer detection by screening-presenting circulating miRNAs as a promising next generation biomarker breakthrough. Asian Pac J Cancer Prev 14 2167-2172, 2013

3. Naruke T, Goya T, Tsuchiya R and Suemasu K: Prognosis and survival in resected lung carcinoma based on the new internationa staging system. J Thorac Cardiovasc Surg 96: 440-447, 1988.

4. Ginsberg RJ and Rubinstein LV: Randomized trial of lobectomy versus limited resection for T1 N0 non-small cell lung cancer. Lung Cancer Study Group. Ann Thorac Surg 60: 615-622; discussion 622-623, 1995.

5. Goldstraw P, Crowley J, Chansky K, Giroux DJ, Groome PA, Rami-Porta R, Postmus PE, Rusch V and Sobin L; International Association for the Study of Lung Cancer International Staging Committee; Participating Institutions: The IASLC Lung Cancer Staging Project: Proposals for the revision of the TNM stage groupings in the forthcoming (seventh) edition of the TNM Classification of malignant tumours. J Thorac Oncol 2: 706-714, 2007.

6. Lu X, Yang X, Zhang Z and Wang D: Meta-analysis of serum tumor markers in lung cancer. Zhongguo Fei Ai Za Zhi 13: 1136-1140 (In Chinese)

7. Dennis G Jr, Sherman BT, Hosack DA, Yang J, Gao W, Lane HC and Lempicki RA: DAVID: Database for annotation, visualization, and integrated discovery. Genome Biol 4: P3, 2003.

8. Ashburner M, Ball CA, Blake JA, Botstein D, Butler $\mathrm{H}$, Cherry JM, Davis AP, Dolinski K, Dwight SS, Eppig JT, et al: Gene Ontology: Tool for the unification of biology. The Gene Ontology Consortium. Nat Genet 25: 25-29, 2000.

9. Kanehisa M and Goto S: KEGG: Kyoto encyclopaedia of genes and genomes. Nucleic Acids Res 28: 27-30, 2000.

10. Sanchez-Palencia A, Gomez-Morales M, Gomez-Capilla JA, Pedraza V, Boyero L, Rosell R and Fárez-Vidal ME: Gene expression profiling reveals novel biomarkers in nonsmall cell lung cancer. Int J Cancer 129: 355-364, 2011.

11. Rousseaux S, Debernardi A, Jacquiau B, Vitte AL, Vesin A, Nagy-Mignotte H, Moro-Sibilot D, Brichon PY, Lantuejoul S, Hainaut $\mathrm{P}$, et al: Ectopic activation of germline and placental genes identifies aggressive metastasis-prone lung cancers. Sci Transl Med 5: 186ra66, 2013.

12. Meister M, Belousov A, Xu EC, Schnabel P, Warth A and Hoffman $\mathrm{H}$ : Intra-tumor heterogeneity of gene expression profiles in early stage non-small cell lung cancer. J Bioinf Res Stud 1: 1, 2014.

13. Barrett T, Wilhite SE, Ledoux P, Evangelista C, Kim IF, Tomashevsky M, Marshall KA, Phillippy KH, Sherman PM, Holko M, et al: NCBI GEO: Archive for functional genomics data sets-update. Nucleic Acids Res 41: D991-D995, 2013.

14. Franceschini A, Szklarczyk D, Frankild S, Kuhn M, Simonovic M, Roth A, Lin J, Minguez P, Bork P, von Mering C and Jensen LJ: STRING v9.1: Protein-protein interaction networks, with increased coverage and integration. Nucleic Acids Res 41: D808-D815, 2013.
15. Shannon P, Markiel A, Ozier O, Baliga NS, Wang JT, Ramage D, Amin N, Schwikowski B and Ideker T: Cytoscape: A software environment for integrated models of biomolecular interaction networks. Genome Res 13: 2498-2504, 2003.

16. Bader GD and Hogue CW: An automated method for finding molecular complexes in large protein interaction networks. BMC Bioinformatics 4: 2, 2003.

17. Győrffy B, Surowiak P, Budczies J and Lánczky A: Online survival analysis software to assess the prognostic value of biomarkers using transcriptomic data in non-small-cell lung cancer. PLoS One 8: e82241, 2013.

18. Jakopovic M, Thomas A, Balasubramaniam S, Schrump D, Giaccone $G$ and Bates SE: Targeting the epigenome in lung cancer: Expanding approaches to epigenetic therapy. Front Oncol 3: 261, 2013.

19. Singhal S, Vachani A, Antin-Ozerkis D, Kaiser LR and Albelda SM: Prognostic implications of cell cycle, apoptosis, and angiogenesis biomarkers in non-small cell lung cancer: A review. Clin Cancer Res 11: 3974-3986, 2005.

20. Voortman J, Checińska A and Giaccone AG: The proteasomal and apoptotic phenotype determine bortezomib sensitivity of non-small cell lung cancer cells. Mol Cancer 6: 73, 2007.

21. Yang Y, Ikezoe TT, Saito T, Kobayashi M, Koeffler HP and Taguchi H: Proteasome inhibitor PS-341 induces growth arrest and apoptosis of non-small cell lung cancer cells via the JNK/c-Jun/AP-1 signaling. Cancer Sci 95: 176-180, 2004.

22. Huang CL, Yokomise $\mathrm{H}$ and Miyatake A: Clinical significance of the p53 pathway and associated gene therapy in non-small cell lung cancers. Future Oncol 3: 83-93, 2007.

23. Kastan MB and Bartek J: Cell-cycle checkpoints and cancer. Nature 432: 316-323, 2004.

24. Malumbres M and Barbacid M: Mammalian cyclin-dependent kinases. Trends Biochem Sci 30: 630-641, 2005.

25. Wang Q, Su L, Liu N, Zhang L, Xu W and Fang H: Cyclin dependent kinase 1 inhibitors: A review of recent progress. Curr Med Chem 18: 2025-2043, 2011.

26. Sharma PS, Sharma R and Tyagi R: Inhibitors of cyclin dependent kinases: Useful targets for cancer treatment. Curr Cancer Drug Targets 8: 53-75, 2008.

27. Malumbres M, Pevarello P, Barbacid M and Bischoff JR: CDK inhibitors in cancer therapy: What is next? Trends Pharmacol Sci 29: 16-21, 2008.

28. Yim H: Current clinical trials with polo-like kinase 1 inhibitors in solid tumors. Anticancer Drugs 24: 999-1006, 2013.

29. Baumann P and West SC: Role of the human RAD51 protein in homologous recombination and double-stranded-break repair. Trends Biochem Sci 23: 247-251, 1998.

30. Venkitaraman AR: Cancer susceptibility and the functions of BRCA1 and BRCA2. Cell 108: 171-182, 2002.

31. Raderschall E, Stout K, Freier S, Suckow V, Schweiger S and Haaf T: Elevated levels of Rad51 recombination protein in tumor cells. Cancer Res 62: 219-225, 2002.

32. Davies AA, Masson JY, McIlwraith MJ, Stasiak AZ, Stasiak A, Venkitaraman AR and West SC: Role of BRCA2 in control of the RAD51 recombination and DNA repair protein. Mol Cell 7: 273-282, 2001.

33. Johnson A, Yao NY, Bowman GD, Kuriyan J and O'Donnell M: The replication factor $\mathrm{C}$ clamp loader requires arginine finger sensors to drive DNA binding and proliferating cell nuclear antigen loading. J Biol Chem 281: 35531-35543, 2006.

34. Kim HS and Brill SJ: Rfc4 interacts with Rpal and is required for both DNA replication and DNA damage checkpoints in Saccharomyces cerevisiae. Mol Cell Biol 21: 3725-3737, 2001.

35. Xiang J, Fang L, Luo Y, Yang Z, Liao Y, Cui J, Huang M, Yang Z, Huang Y, Fan X, et al: Levels of human replication factor $\mathrm{C} 4$, a clamp loader, correlate with tumor progression and predict the prognosis for colorectal cancer. J Transl Med 12: 320, 2014.

36. Lin J, Marquardt G, Mullapudi N, Wang T, Han W, Shi M, Keller S, Zhu C, Locker J and Spivack SD: Lung cancer transcriptomes refined with laser capture microdissection. Am J Pathol 184: 2868-2884, 2014.

37. Hallou A, Jennings $\mathbf{J}$ and Kabla AJ: Tumour heterogeneity promotes collective invasion and cancer metastatic dissemination. R Soc Open Sci 4: 161007, 2017.

38. Fend F and Raffeld M: Laser capture microdissection in pathology. J Clin Pathol 53: 666-672, 2000.

This work is licensed under a Creative Commons Attribution-NonCommercial-NoDerivatives 4.0 International (CC BY-NC-ND 4.0) License. 